\title{
Calculation and experiment of spiral bevel gear by duplex helical method predesigned contact path
}

\author{
Longlong GENG*, Xiaozhong DENG**, Jing DENG**, Chuang JIANG*** and Zhengyang HAN* \\ *School of Mechatronic Engineering, Northwestern Polytechnical University, \\ 127 Youyi West Rd, Xi'an 710072, China \\ ${ }^{* *}$ Collaborative Innovation Center of Machinery Equipment Advanced Manufacturing, Henan Province, \\ 48 Xiyuan Rd, Luoyang 471003, China \\ *** School of Mechatronics Engineering, Henan University of Science and Technology, \\ 48 Xiyuan Rd, Luoyang 471003, China \\ E-mail: gll0379@haust.edu.cn
}

Received: 9 July 2020; Revised: 11 November 2020; Accepted: 12 January 2021

\begin{abstract}
In this paper a method to calculate machine tool setting parameters for spiral bevel gars by duplex helical method is proposed, in which contact path is predesigned. Firstly, machine setting parameters and curvature parameters in reference points of gear are calculated; secondly, curvature parameters of technology and cut in reference points of pinion are calculated based on meshing theory; then, machine tool setting parameters of pinion are calculated on the basic of the relationship of curvature parameters of technology and cut. Mathematic models of tooth contact analysis and ease-off topology are established to check out the machine tool setting parameters. An example is presented that machine tool setting parameters are calculated based on the method proposed in this paper, which predesign contact paths are $45^{\circ}, 60^{\circ}$, and $70^{\circ}$ respectively. The contact path of TCA results exist errors are less than $0.5 \%$ comparing to predesign; the results achieve the design requirement. Finally a cut experiment is carried out which the predesign of contact path is $70^{\circ}$; the results of rolling test are consistent with results of TCA. The correctness and validity of the method is verified.
\end{abstract}

Keywords: Spiral bevel gear, Duplex helical method, Predesign contact path, Tooth contact analysis, Experimental verification

\section{Introduction}

Spiral bevel gears are widely used in vehicles, aircrafts, and industrial gearboxes, transmitting rotation between intersecting axes for its advantages of driving smoothly, high transmission efficiency, excellent load capacity, etc. It is essential to calculate machine tool setting parameters to process spiral bevel gears. Traditionally, machine setting parameters are calculated by local conjugate method or local synthesis method. The machine setting parameters of pinion concave side and convex side are calculated according to machine setting parameters of gear separately. In order to get the correct curvature relation between gear and pinion which meet the requirements of meshing performance, a tooth length curvature correction is introduced during machine-tool setting parameters of pinion are calculated. While the correction of tooth length curvature is obtained by adjusting the cut radius, that is, increasing the radius of inner cut and reducing the radius of outer cut, which lead to the result that the radius of internal cut is larger than that of external cut. So it cannot be installed on the same cutter. Therefore, the traditional method is also known as five-cut method, these are rough cut for gear, finish cut for gear, rough cut for pinion, finish cut for concave side of pinion, and finish cut of convex side. It has studied by many experts and scholars. Generalized theory and methods of spiral bevel and hypoid gears manufactured by the five-cut process have been comprehensively presented by several gear scientists (1988, 2006, and 2011). Shtipelman (1978) introduced the generalized theory of the five-cut process and calculated machine settings parameters of spiral bevel and hypoid gears by the five-cut process in Gleason Works. Litvin (1991, 2004) proposed 
local synthesis and applied for tooth contact analysis (TCA), and determined the optimal machine settings parameters.

Recently great changes have taken place in the way of processing method that duplex helical method $(1971,1981)$ is gradually replacing five-cut method with the development of computers and CNC machine tools. In this method, concave side and convex side for both gear and pinion are both processed simultaneously by a cutter. Tilt and helical motion are introduced to modify the tooth length curvature for both pinion and gear to solve the curvature change caused by the inner and outer cut being mounted on the same cutter. It is more efficient and energy-saving and environmental protection by adopt power dry cutting. Obviously, duplex helical method is an advanced method and further research is necessary and essential.

Duplex helical method is firstly proposed by Gleason (1971) to machine spiral bevel gear, while cutting principle and machine setting parameters are not fully revealed. Gonzalez et al. (2011) approached conversion of the specific machine-tool settings of a given generator to neutral machine-tool setting by duplex heliacal method; Tsay et al., (1993) developed a mathematical model can be applied to simulate the tooth surface geometry machined by duplex spread blade and duplex helical method; however the calculation of machine-tool settings are not involved in above literatures. Until recently, Zhang et al., (2016) proposed a methodology for calculating the basic machine-tool settings for spiral bevel and hypoid gears by duplex helical method in which gear is non-generated, while contact characteristic are not designed.

Contact characteristic is one of the important bases to evaluate the performance of transmission. Many researches have been studied in active design and modification of tooth surface for spiral bevel gears to optimize contact performance. Litvin F.L. (1991) proposed local synthesis method for five-cut method which the meshing performance is optimized with the help of controllability of the second order contact parameters; Cao Xuemei and Fang Zongde (2007) proposed a method designing pinion surface based on a predesigned parabolic function of transmission errors with limited magnitude of maximal transmissions errors and a predesigned liner function of contact path. While no similar literatures for Duplex helical method. Ship (2010) proposed a novel ease-off flank modification methodology for spiral bevel and hypoid gears to predesigned transmission error and contact line, Nie shaowu (2018) proposed a mismatch topography adjustment method of tooth surface modification to improve the meshing performance. Above literatures tend to solve the machine-tool settings according to the ease-off modification to optimize contact performance and the relation between ease-off surfaces modification and contact properties is not revealed. Zhang weiqing (2018) established a correlation model between the contact area morphological parameters and the ease-off tooth surface modification by using the relative curvature and line conjugate surface characteristics realized the mesh performance control.

As the machine-tool setting parameters are calculated, tooth contact analysis is traditional tool to check spiral bevel gear mesh performance. Litvin F.L. (1991) presented the tooth contact analysis in detail; Fan Qi (2007) proposed a modified algorithm of tooth contact simulation with reduced number of equations of the nonlinear iterations and stabilized iteration convergence Cao xuemei (2018) proposed a new method for gear tooth contact analysis solved two considerable disadvantages of the generalized algorithm. It is a trend that ease-off topography is used as a visual tool for analysis and synthesis of contact characteristics. M. Kolivand (2010) proposed establishment of ease-off topography based on tooth surface deviations and used to perform tooth contact analysis. Fan Qi (2016) described the theory of ease-off and applied in tooth contact analysis with complex tooth surface modifications.

On the basis of previous scholars' researches, this paper proposed a method to process spiral bevel gear by duplex helical method which predesigned contact path. Concave side and convex side for both gear and pinion are both processed simultaneously. Therefore, alternate cutter is used in finish cut of pinion in this method while inner finish cutter and outer finish cutter are used in finish cut of pinion in five-cut method. Tilt motion is introduced to the process for both gear and pinion to modify the tooth length curvature. And a helical motion is also introduced to process of pinion which is not used in five-cut method. The machine-tool setting parameters are calculated according to curvature relation, and then tooth contact analysis and ease-off topography model are established to check the machine tool setting parameters.

\section{Calculate machine-tool setting parameters 2.1 Technical route}

The machine setting parameters are calculated based on the meshing equation in the reference points located in 
concave side, convex side and root, respectively. In this paper, the contact path is defined as an angle between the tangent direction of contact path and perpendicular of root in gear. In particular, the angle of contact path is predesigned by calculating relative curvatures along the direction of contact path and its perpendicular. And the technical route is listed in Fig.1. The first subscript (1or2) represents pinion or gear; t he second subscript (1or2) is a representation of convex side or concave side. For example, $\mathbf{r}_{21}$ represents the tooth surface of gear convex side, $r_{21}$ represents cut radius of gear convex side.
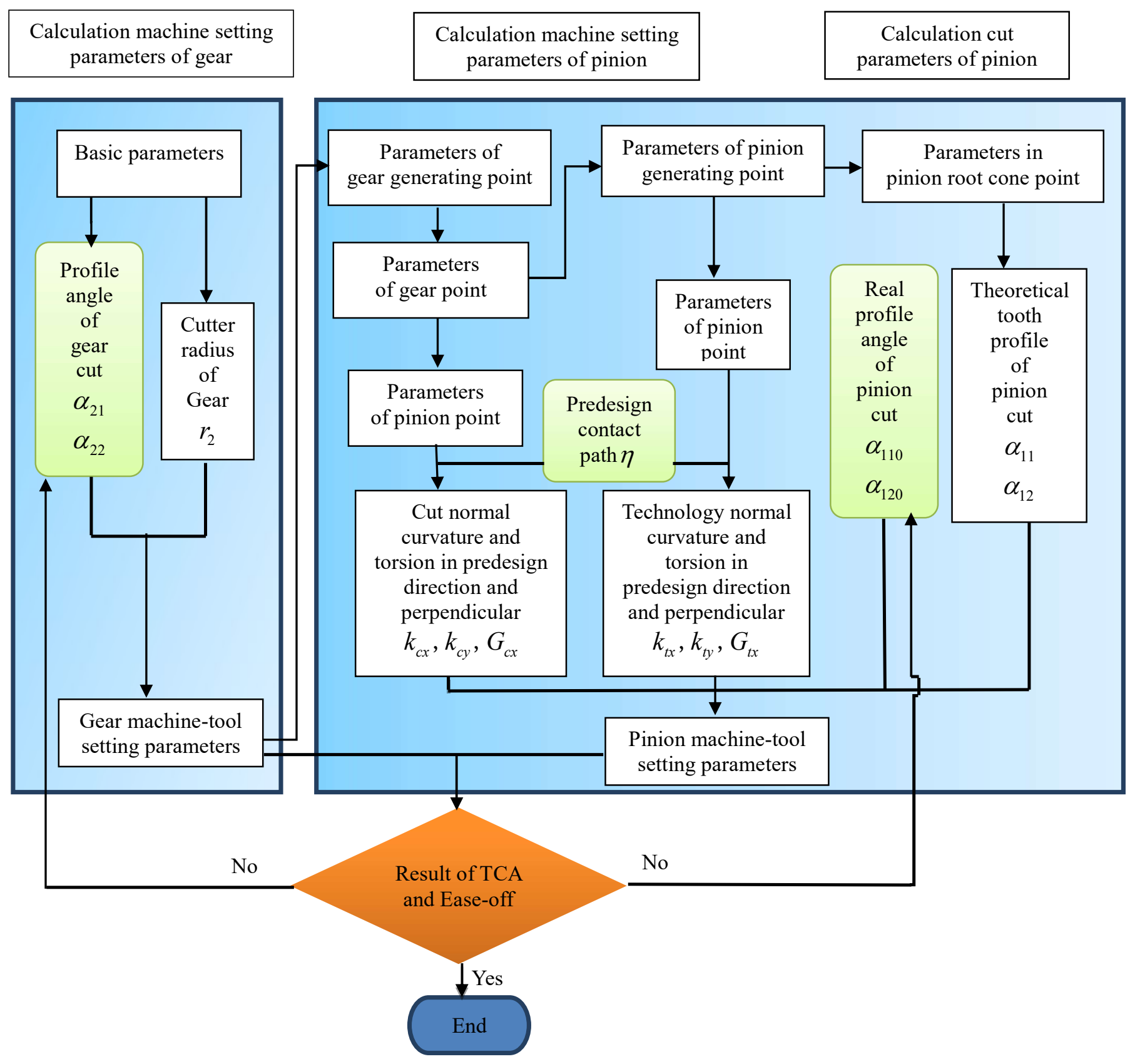

Fig.1 Flowchart of technology route

\subsection{Machine settings parameters of gear}

Installment of gear process is showed in Fig.2; root cone of gear is perpendicular to the axis of cradle. In order to calculate machine setting parameters of gear, firstly parameters of pitch cone for gear and gear generating are obtained as follow. 


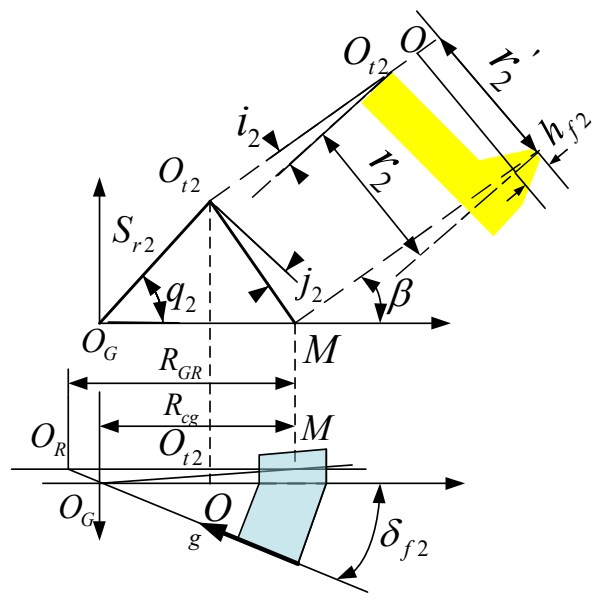

Fig.2 Calculation of gear machine parameters

The spiral angle $\beta_{G R}$, pressure angle $\alpha_{G R}$, cone distance $R_{G R}$ of gear root cone can be derived as

$$
\left\{\begin{array}{c}
\beta_{G R}=\beta \\
\sin \alpha_{G R}=\cos \theta_{f 2} \sin \alpha+\sin \theta_{f 2} \cos \alpha \sin \beta \\
R_{G R}=R_{e} \frac{\sin \delta_{2}}{\sin \delta_{f 2}}
\end{array}\right.
$$

And the spiral angle $\beta_{c g}$, cone distance $R_{c g}$, offset $E_{c g}$ of generating pitch cone can be derived as

$$
\left\{\begin{array}{l}
\beta_{c g}=\beta \\
R_{c g}=R_{e} \cos \theta_{f 2} \\
E_{c g}=0
\end{array}\right.
$$

where, $\alpha$ is pressure angle, $\beta$ is spiral angle, $\delta_{2}$ is gear pitch angle, $\delta_{f 2}$ is gear root angle, $\theta_{f 2}$ is gear dedendum angle, and $R_{e}$ is cone distance.

Theoretically, profile angle $\alpha_{21}$ of gear cut should be equal to $\alpha_{G R}$, while a small difference can be compensated by tilting axis of gear cut, as Fig. 2 showed, and tilt angle can be calculated as

$$
i_{2}=\alpha_{21}-\alpha_{G R}
$$

where, an explanation is that the tilt angle $i_{2}$ is calculated based on convex side of gear.

For spiral bevel gear, apex of pitch cone is on the line of cradle axis, so

$$
\left\{\begin{array}{l}
E_{m 2}=0 \\
X_{g 2}=0
\end{array}\right.
$$

The distance between $O_{t 2}$ and mounting surface can be derived as $O_{t 2} O$

$$
h=r_{2} \sin i_{2}-h_{f 2}
$$

where, $r_{2}$ is gear cut radius, $r_{2} \cos i_{2}$ is projection into machine plane, $r_{2} \sin i_{2}$ is projection into axis of cut, as shown in Fig.2, $h_{f 2}$ is dedendum of gear.

Then sliding base can be $X_{B 2}$ can be derived as

$$
X_{B 2}=h+\left(\frac{R_{G R}}{R_{c g}}\right) \tan \delta_{f 2}
$$

Cradle angle can be derived as 
$\tan q_{2}=\frac{r_{2} \cos i_{2} \cos \beta}{R_{c g}-r_{2} \cos i_{2} \sin \beta}$

Swivel angle be derived as

$j_{2}=q_{2}-\beta$

Radial setting can be derived as

$S_{r 2}=\frac{r_{2} \cos i_{2} \cos \beta}{\sin q_{2}}$

Roll ratio can be derived as

$R_{a g}=\frac{\cos \theta_{f 2}}{\sin \delta_{2}}$

\subsection{Machine settings parameters of pinion}

Machine-tool setting parameters of pinion are calculated based on that of gear. According to the gear machine setting parameters, curvature of reference points on convex side and concave side in gear generating can be deduced, and then curvature of gear and pinion can be obtained which are line conjugate surface between meshing surfaces. In order to realize point contact, a certain direction of tangent to contact path is predesigned, then technology curvature of pinion along direction of contact path and its perpendicular can be calculated; cut curvature of pinion can be deduced according to coordinate of pinion processing and machine-tool setting parameters of pinion; finally machine-tool setting parameters of pinion can be calculated according to equality of the corresponding cut and technology curvature.

\subsubsection{Technology curvature of pinion}

Mathematical model of gear process is showed in Fig.3, position vector $\mathbf{r}_{t}$, normal vector $\mathbf{n}_{t}$ and tangent vector $\boldsymbol{\tau}_{t}$ of the reference point in coordinate system of cut can be expressed as

$$
\left\{\begin{array}{l}
\mathbf{r}_{\mathbf{t}}=f(u, \theta) \\
\boldsymbol{\tau}_{\mathbf{t}}=f(u, \theta) \\
\mathbf{n}_{\mathbf{t}}=f(u, \theta)
\end{array}\right.
$$

where, $u, \theta$ are parameters of tooth surface.

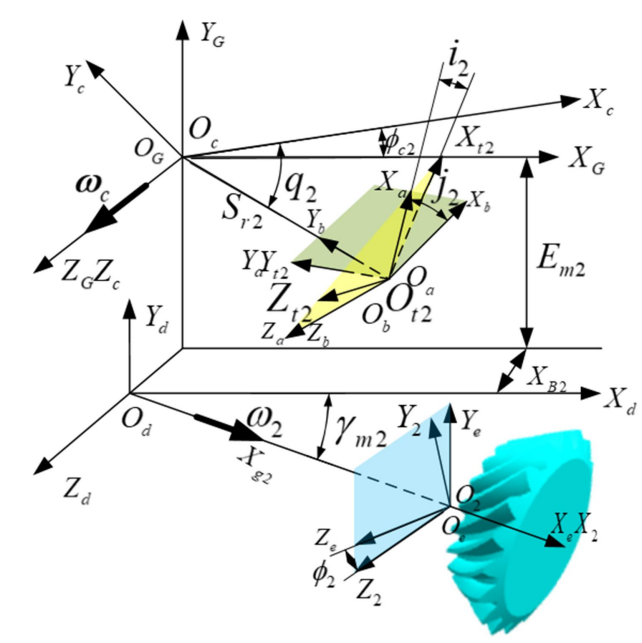

Fig.3 Coordinate system of gear process 
The above vectors represented in coordinate system of gear generating $S_{G}$ and gear $S_{2}$ can be expressed as

$$
\left\{\begin{array}{l}
\mathbf{r}_{\mathbf{G} \mathbf{2}}=M_{G c} M_{c b} M_{b a} M_{a t 2} \mathbf{r}_{\mathbf{t}} \\
\mathbf{n}_{\mathbf{G} \mathbf{2}}=L_{G c} L_{c b} L_{b a} L_{a t 2} \mathbf{n}_{\mathbf{t}} \\
\boldsymbol{\tau}_{\mathbf{G} \mathbf{2}}=L_{G c} L_{c b} L_{b a} L_{a t 2} \boldsymbol{\tau}_{\mathbf{t}} \\
\mathbf{r}_{\mathbf{2}}=M_{2 e} M_{e d} M_{d G} \mathbf{r}_{\mathbf{G} \mathbf{2}} \\
\mathbf{n}_{\mathbf{2}}=L_{2 e} L_{e d} L_{d G} \mathbf{n}_{\mathbf{G} \mathbf{2}} \\
\boldsymbol{\tau}_{\mathbf{2}}=L_{2 e} L_{e d} L_{d G} \boldsymbol{\tau}_{\mathbf{G} \mathbf{2}}
\end{array}\right.
$$

where, $M$ is transformation matrix, and $L$ is $3 \times 3$ minor of $M$.

Meshing equation is satisfied in reference point and expressed as

$$
f\left(u, \theta, \phi_{c 2}\right)=\mathbf{n}_{2} \cdot \frac{\partial \mathbf{r}_{2}}{\partial \phi_{c 2}}=0
$$

where, $\phi_{c 2}$ is cradle angle during the process of gear.

Rotating projection point of reference point can be expressed as

$$
\left\{\begin{array}{l}
X L=\mathbf{r}_{2}(1) \\
R L^{2}=\mathbf{r}_{2}^{2}(2)+\mathbf{r}_{2}^{2}(3)
\end{array}\right.
$$

By solving Eq. (13) and Eq. (14), tooth surface parameters $u, \theta$ and $\phi_{c 2}$ can be deduced, plug it into Eq. (12) and Eq. (13), the relative vectors are obtained.

Above calculation can be applied to both concave side and convex side for gear. And convex side is used as an illustration in the following. The curvature and torsion of reference point in gear generating can be expressed as

$$
\left\{\begin{array}{l}
K_{c g x 1}=\frac{\cos a_{21}}{r_{21}-u \sin a_{21}} \\
K_{c g y 1}=0 \\
G_{c g x 1}=0
\end{array}\right.
$$

where, $r_{21}$ is cut radius of gear convex side, $K_{c g x 1}$ is curvature along $\boldsymbol{\tau}_{21} \times \mathbf{n}_{21}, K_{c g y 1}$ is curvature along $\boldsymbol{\tau}_{21}$ and $G_{c g x 1}$ is torsion along $\boldsymbol{\tau}_{21} \times \mathbf{n}_{21}$. $G_{g x 1}$.

Then curvature and torsion of reference in gear can be calculated (Litvin, F.L.,2004), and represented as $K_{g x 1} 、 K_{g y 1}$ 、

Assumption that an angle $\theta_{G}$ is take place for gear. After rotation, the reference point obtained by Eq. (12) is contact point between gear and pinion, and the vectors can be expressed as

$$
\left\{\begin{array}{l}
\mathbf{r}_{11}=\mathbf{r}_{21}=L\left[g, \theta_{G}\right] \mathbf{r}_{21} \\
\mathbf{n}_{11}=\mathbf{n}_{21}=L \mathbf{r}_{21} \\
\boldsymbol{\tau}_{11}=\boldsymbol{\tau}_{21}=L \boldsymbol{\tau}_{21}
\end{array}\right.
$$

Meshing equation between pinion and gear can be expressed as

$$
\begin{aligned}
& \mathbf{n}_{21} \cdot \mathbf{v}_{G P}=0 \\
& \left\{\begin{array}{l}
\boldsymbol{\omega}_{G}=\left[-\cos \gamma_{m 2}, 0, \sin \gamma_{m 2}\right] \\
\boldsymbol{\omega}_{p}=\left[-\sin \gamma_{m 2}, 0,-\cos \gamma_{m 2}\right] \\
\boldsymbol{\omega}_{G P}=\boldsymbol{\omega}_{G}-\boldsymbol{\omega}_{P} \\
\mathbf{v}_{G P}=\left(\boldsymbol{\omega}_{G}-\boldsymbol{\omega}_{P}\right) \times \mathbf{r}_{11}
\end{array}\right.
\end{aligned}
$$

By solving Eq. (17), the rotate angle $\theta_{G}$ can be obtained, plug it into Eq. (16), the vectors in coordinate system of pinion concave side can be obtained and represented as position vector $\mathbf{r}_{11}$, normal vector $\mathbf{n}_{11}$ and tangent vector $\boldsymbol{\tau}_{11}$. Similarly, curvature and torsion of reference point can be calculated, and represented as $K_{p t x 1} 、 K_{p t y 1}$ 、 $G_{p t x 1}$. 
The curvature calculated above is on the bias of line contact, and in order to get a point contact in the mesh point, an angle $\eta$ that determines the direction of contact path in gear convex side is chose (Yan Zhida, 1980). Then in pinion concave side, an angle $\Delta \theta$ formed between tangent direction of contact path $\mathbf{u}_{11}$ and $\boldsymbol{\tau}_{11} \times \mathbf{n}_{11}$, as shows in Fig.4.

$$
\tan \Delta \theta=-\frac{\Delta K_{p y}+\Delta G_{p x} \tan \eta}{\Delta G_{p x}+\Delta K_{p x} \tan \eta}
$$

where, $\Delta K_{p x} \Delta K_{p y} \Delta G_{p x}$ are relative curvature (Litvin, F.L., 2004) in the reference point between pinion and gear along the direction of $\boldsymbol{\tau}_{11} \times \mathbf{n}_{11}$ and $\boldsymbol{\tau}_{11}$.

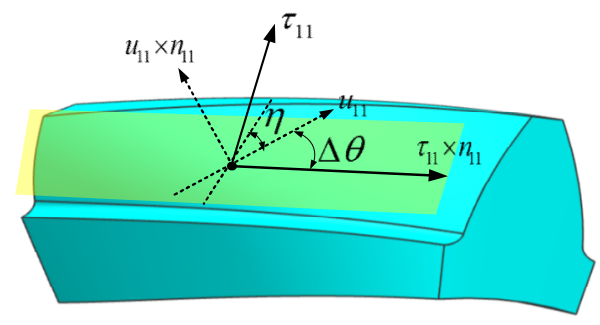

Fig.4 Direction of contact path in pinion tooth surface

Tangent direction of contact path $\mathbf{u}_{11}$ can be expressed as

$\mathbf{u}_{11}=\boldsymbol{\tau}_{11} \times \mathbf{n}_{11} \sin \Delta \theta+\boldsymbol{\tau}_{11} \cos \Delta \theta$

On the basis of Euler and Bertrand Equation, the curvature and torsion in direction of $\mathbf{u}_{11} \times \mathbf{n}_{11}$ and $\mathbf{u}_{11}$ can be calculated and represented as $K_{t x 1} 、 K_{t y 1} 、 G_{t x 1}$

$$
\left\{\begin{array}{l}
K_{t x 1}=K_{p t x 1} \cos ^{2} \Delta \theta-2 G_{p t x 1} \sin \Delta \theta \cos \Delta \theta+K_{p t y 1} \sin ^{2} \Delta \theta \\
K_{t y 1}=K_{p t x 1} \cos ^{2} \Delta \theta+2 G_{p t x 1} \sin \Delta \theta \cos \Delta \theta+K_{p t y 1} \sin ^{2} \Delta \theta \\
G_{t x 1}=G_{p t x 1}\left(\cos ^{2} \Delta \theta-\sin ^{2} \Delta \theta\right)+\left(K_{p t x 1}-K_{p t y 1}\right) \sin \Delta \theta \cos \Delta \theta
\end{array}\right.
$$

Similarly, convex side reference point of pinion can be obtained, and represented as position vector $\mathbf{r}_{12}$, normal vector $\mathbf{n}_{12}$, tangent direction of contact path $\mathbf{u}_{12}$, curvature and torsion $K_{t x 2} 、 K_{t y 2} 、 G_{t x 2}$ in direction of $\mathbf{u}_{12} \times \mathbf{n}_{12}$ and $\mathbf{u}_{12}$.

As the reference points selected are in two side of gear tooth ace, so corresponding points calculated by Eq. (16) are in two side of tooth. For convenience a rotation $\theta_{1}$ is introduced. After rotate, the position vector $\mathbf{r}_{11}$ and $\mathbf{r}_{12}$ are in the two side of pinion tooth ace, and the angle $\theta_{1}$ can be determined as

$$
\left\{\begin{array}{l}
\theta_{1}=\frac{2 \pi+\left(\theta_{G 1}-\theta_{G 2}+B_{\text {min }} / R_{e}\right)}{Z_{1}} \\
\mathbf{A}=L\left[p, \theta_{1}\right] \mathbf{A}
\end{array}\right.
$$

Where, A represent position vector $\mathbf{r}_{12}$ and normal vector $\mathbf{n}_{12}$ and tangent direction of contact path $\mathbf{u}_{12} ; Z_{1}$ is number of pinion, $B_{\min }$ is clearance, $\theta_{G 1} 、 \theta_{G 2}$ can be obtained by Eq.(17).

\subsubsection{Cut curvature of pinion}

The process of pinion is on the base of Fig.2, and the points in concave side and convex side of pinion corresponding that of gear are known and represented as position vector $\mathbf{r}_{11} 、 \mathbf{r}_{12}$, normal vector $\mathbf{n}_{11} 、 \mathbf{n}_{12}$, respectively.

As Fig.2 shown, axis of pinion can be expressed as $\mathbf{p}=\left[\begin{array}{llll}-\sin \gamma_{m 2} & 0 & -\cos \gamma_{m 2}\end{array}\right]$. Axis of pinion generating $\mathbf{c}_{p}$ can be expressed as

$\mathbf{c}_{p}=m_{1} \mathbf{n}_{11}+m_{2} \mathbf{p}+m_{3}\left(\mathbf{n}_{11} \times \mathbf{p}\right)$

The pressure angle of reference point in pinion generating can be expressed as 
$\sin \alpha_{c 1}=-\left(\mathbf{c}_{p} \cdot \mathbf{n}_{11}\right)$

The machine root angle can be expressed as

$\sin \gamma_{m 1}=-\left(\mathbf{c}_{p} \times \mathbf{p}\right)$

Then Eq. (23) can be simplified as

$\mathbf{c}_{p}=\mathbf{a}_{\mathrm{T} 1}-m \mathbf{a}_{\mathrm{T} 2}$

where $\quad \mathbf{a}_{\mathrm{T} 1}=m_{1} \mathbf{n}_{11}+m_{2} \mathbf{p} \quad, \quad \mathbf{a}_{\mathrm{T} 2}=\mathbf{n}_{11} \times \mathbf{p} \quad, \quad m=\sqrt{\frac{1-\mathbf{a}_{\mathrm{T} 1} \bullet \mathbf{a}_{\mathrm{T} 1}}{\left(\mathbf{n}_{11} \bullet \mathbf{p}\right)^{2}}} \quad, \quad m_{1}=\frac{-\sin \alpha_{c 1}+\sin \gamma_{m 1}\left(\mathbf{n}_{11} \bullet \mathbf{p}\right)}{1-\left(\mathbf{n}_{11} \bullet \mathbf{p}\right)^{2}} \quad$, $m_{2}=\frac{-\sin \gamma_{m 1}+\sin \alpha_{c 1}\left(\mathbf{n}_{11} \bullet \mathbf{p}\right)}{\left(\mathbf{n}_{11} \bullet \mathbf{p}\right)^{2}}$

Base on the axis of pinion generating $\mathbf{c}_{p}$ and the axis of pinion $\mathbf{p}$, coordinate $\operatorname{system}\left(\mathbf{e}_{p} \times \mathbf{c}_{p} 、 \mathbf{e}_{p} 、 \mathbf{c}_{p}\right)$ of pinion process can be established as Fig.5, thereinto $\mathbf{e}_{p}=\mathbf{p} \times \mathbf{c}_{p}$ and origin of coordinates $O_{p}$ is coincide with $O_{G}$.As Fig.5 shown, machine plane is across $\mathbf{e}_{p} \times \mathbf{c}_{p}$ and perpendicular to $\mathbf{c}_{p}$, distance between machine plane and cross point of $\mathbf{p}$ and $\mathbf{c}_{p}$ is sliding base $X_{B 1}$, distance between the cross point and design point $O_{1}$ is machine center to cross point $X_{g 1}$.

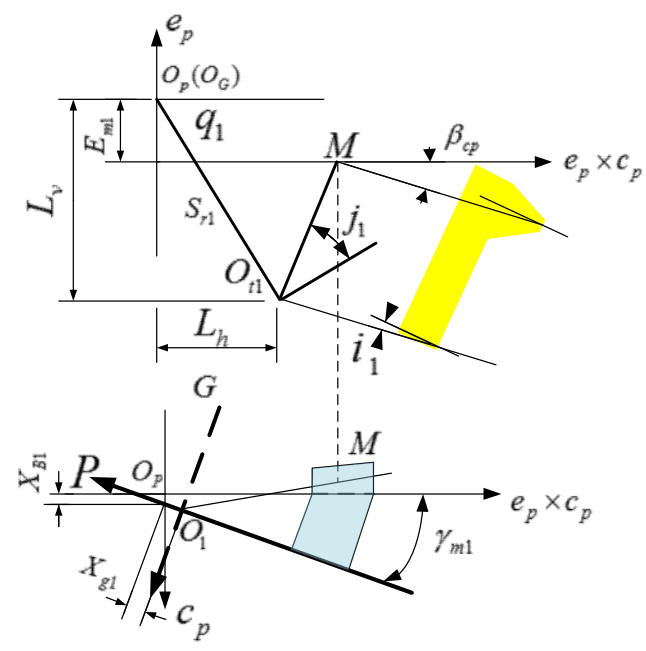

Fig.5 Coordinate system of process pinion

Then position vector and normal vector in concave side of pinion generating (red color in Fig.6) can be expressed

$$
\left\{\begin{array}{l}
\mathbf{A}_{11}=\mathbf{r}_{11}-\left(E_{m 1} \cdot \mathbf{e}_{p}+X_{g 1} \cdot \mathbf{p}\right) \\
\mathbf{N}_{11}=\mathbf{n}_{11}
\end{array}\right.
$$

where, $\mathbf{A}_{11}$ is position vector and $\mathbf{N}_{11}$ is normal vector in pinion generating. It should be clearly noted that the influence of $X_{B 1}$ is not considered.

Meshing equation is satisfied between the pinion and pinion generating in reference point can be expressed as

$$
\left\{\begin{array}{l}
\mathbf{V}_{p c 1} \cdot \mathbf{N}_{11}=0 \\
\mathbf{V}_{p c 1}=\left(\begin{array}{lll}
R_{a p} & \mathbf{p} & \mathbf{r}_{11}
\end{array}\right)+\left(\mathbf{p} \times \mathbf{A}_{11}\right)+H \cdot \mathbf{c}_{p}
\end{array}\right.
$$

where, $\mathbf{V}_{p c 1}$ is relative velocity between pinion and pinion generating in reference point and $H$ is helical motion velocity coefficient.

Solving Eq.(28), roll ratio $R_{a p}$ can be expressed as

$$
R_{a p}=f\left(\alpha_{c 1}, \gamma_{m 1}, X_{g 1}, E_{m 1}, H\right)
$$

Similarly position vector and normal vector in convex side of pinion generating (green color in Fig.6) can be expressed 


$$
\left\{\begin{array}{l}
\mathbf{A}_{12}=\mathbf{r}_{12}-\left(E_{m 1} \cdot \mathbf{e}_{p}+X_{g 1} \cdot \mathbf{p}\right) \\
\mathbf{N}_{12}=\mathbf{n}_{12}
\end{array}\right.
$$

A ration angle $\theta_{p}$ takes place for pinion in process, and the reference point of convex can be expressed as

$$
\left\{\begin{array}{l}
\mathbf{A}_{12}=L\left[p, \theta_{p}\right] \mathbf{A}_{12} \\
\mathbf{N}_{12}=L\left[p, \theta_{p}\right] \mathbf{n}_{12}
\end{array}\right.
$$

Meshing equation is also satisfied between pinion and pinion generating in the reference point.

$$
\left\{\begin{array}{l}
\mathbf{V}_{p c 2} \cdot \mathbf{N}_{12}=0 \\
\mathbf{V}_{p c 2}=\left(\begin{array}{lll}
R_{a p} & \mathbf{p} & \mathbf{r}_{12}
\end{array}\right)+\left(\mathbf{p} \times \mathbf{A}_{12}\right)+H \cdot \mathbf{c}_{p}
\end{array}\right.
$$

By solving Eq. (32), $\theta_{p}$ can be obtained. During process, pinion and pinion generating are rotate in a fixed relation. In order to get position vector and normal vector of convex point in initial position, the pinion generating should be rotate back an angle $\theta_{p} / R_{a p}$, and expressed as

$$
\left\{\begin{array}{l}
\mathbf{A}_{12}=L\left[p,-\frac{\theta_{p}}{R_{a p}}\right] \mathbf{A}_{12}+H\left(-\frac{\theta_{p}}{R_{a p}}\right) \cdot \mathbf{c}_{p} \\
\mathbf{N}_{12}=L\left[p,-\frac{\theta_{p}}{R_{a p}}\right] \mathbf{N}_{12}
\end{array}\right.
$$

According to Eq. (27) and Eq. (33), position vector and normal vector of concave and convex in pinion generating are calculated. Then axis of pinion cut $\mathbf{u}_{p}$ as shown in Fig.6, and can be expressed as

$$
\left\{\begin{array}{l}
\mathbf{A}_{\mathrm{cp} 1}=R_{c 1} \cdot \mathbf{N}_{11}+\mathbf{A}_{11} \\
\mathbf{A}_{\mathrm{cp} 2}=R_{c 2} \cdot \mathbf{N}_{12}+\mathbf{A}_{12} \\
\mathbf{u}_{p}=\frac{\mathbf{A}_{\mathrm{cp} 1}-\mathbf{A}_{\mathrm{cp} 2}}{\left|\mathbf{A}_{\mathrm{cp} 1}-\mathbf{A}_{\mathrm{cp} 2}\right|}
\end{array}\right.
$$

where, $R_{c 1}$ and $R_{c 2}$ are curvature radius corresponding to $\mathbf{A}_{11}$ and $\mathbf{A}_{12}$, as shown in Fig.6.

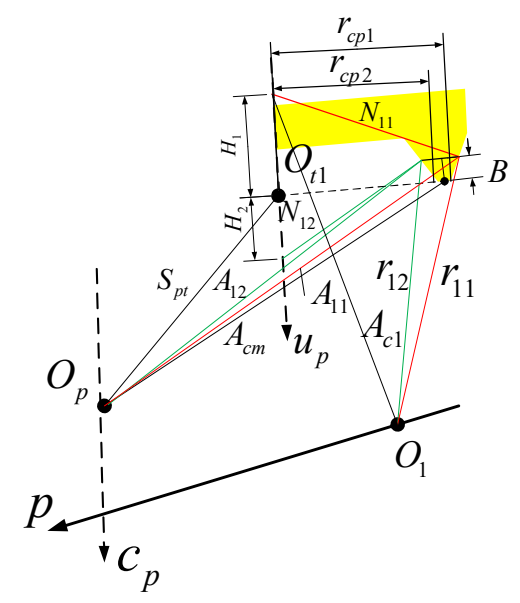

Fig.6 Determine cut parameters of pinion

Suppose $\mathbf{I}_{c 1}=\left(\mathbf{u}_{p} \times \mathbf{n}_{11}\right) /\left|\mathbf{u}_{p} \times \mathbf{n}_{11}\right|$, then a coordinate system $\left\{\mathbf{I}_{c 1}, \mathbf{n}_{11} \times \mathbf{I}_{c 1}, \mathbf{n}_{11}\right\}$ is established in reference point, and curvature and torsion of concave side of pinion generating can be expressed 


$$
\left\{\begin{array}{l}
K_{c p x 1}=\frac{1}{R_{c 1}} \\
K_{c p y 1}=0 \\
G_{c p x 1}=0
\end{array}\right.
$$

where, $K_{c p x 1}$ is curvature along the direction of $\mathbf{I}_{c 1}, K_{c p y 1}$ is curvature along the direction of $\mathbf{n}_{11} \times \mathbf{I}_{c 1}$, and $G_{c p x 1}$ is torsion along the direction that perpendicular to rotation. Then the curvature and torsion in pinion can be calculated and represented as $K_{p c x 1} 、 G_{p c x 1} 、 K_{p c y 1}$.

Angle between tangent direction of contact path $\mathbf{u}_{11}$ and $\mathbf{I}_{c 1}$ can be expressed as

$\sin \delta=\mathbf{u}_{11} \cdot \mathbf{I}_{c 1}$

Then curvature and torsion can be represented in the direction of $\mathbf{u}_{11} \times \mathbf{n}_{11}$ and $\mathbf{u}_{11}$

$$
\left\{\begin{array}{l}
K_{c x 1}=K_{p c x 1} \cos ^{2} \delta-2 G_{p c x 1} \sin \delta \cos \delta+K_{p c y 1} \sin ^{2} \delta \\
K_{c y 1}=K_{p c x 1} \cos ^{2} \delta+2 G_{p c x 1} \sin \delta \cos \delta+K_{p c y 1} \sin ^{2} \delta \\
G_{c x 1}=G_{p c x 1}\left(\cos ^{2} \delta-\sin ^{2} \delta\right)+\left(K_{p c x 1}-K_{p c x 1}\right) \sin \delta \cos \delta
\end{array}\right.
$$

Similarly curvature and torsion of point in pinion convex side can be calculated and represented as $K_{c x 2}$ 、 $K_{c y 2}$ 、 $G_{c x 2}$.

\subsubsection{Machine tool setting parameters of pinion}

Theoretical tooth profile angle of pinion cut can be obtained by

$\left\{\begin{array}{l}\sin \alpha_{11}=-\mathbf{u}_{p} \cdot \mathbf{N}_{11} \\ \sin \alpha_{12}=-\mathbf{u}_{p} \cdot \mathbf{N}_{12}\end{array}\right.$

The curvature and torsion of cut and technology should be equal, and equations are established as follow.

$$
\left\{\begin{array}{l}
K_{c x 1}+K_{c x 2}=K_{t x 1}+K_{t x 2} \\
K_{c y 1}-K_{t y 1}=0 \\
G_{c y 1}-G_{t y 1}=0 \\
K_{c y 2}-K_{t y 2}=0 \\
G_{c y 2}-G_{t y 2}=0 \\
\alpha_{11}-\alpha_{110}=0 \\
\alpha_{12}-\alpha_{120}=0
\end{array}\right.
$$

where, $\alpha_{110}$ and $\alpha_{120}$ are the real profile angle of pinion cut.

By solving Eq. (39), $\gamma_{m 1}, X_{g 1}, E_{m 1}, H$ can be calculated, and $R_{a p}$ can be got by put them into Eq. (29).

As previously mentioned, above parameters are solved that $X_{B 1}$ is not considered. In order to solve the other parameters, another point is selected in the middle of tool nose, as Fig. 6 shows. The position vector presented in pinion generating expressed as

$$
\mathbf{A}_{c m}=\frac{1}{2}\left(\mathbf{r}_{11}+\mathbf{r}_{12}\right)+B \cdot \mathbf{u}_{p}-\left(\mathbf{e}_{p} \cdot E_{m 1}+X_{g 1} \cdot \mathbf{p}\right)
$$

where, $B=h_{f 1}$

The tangent vector perpendicular to cut axis can be expressed as 


$$
\left\{\begin{array}{l}
\mathbf{A}_{c 1}=\mathbf{r}_{11}+R_{c 1} \cdot \mathbf{n}_{11} \\
\boldsymbol{\tau}_{c m}=\frac{\left(\mathbf{A}_{c m}-\mathbf{A}_{c 1}\right) \times \mathbf{u}_{p}}{\left|\left(\mathbf{A}_{c m}-\mathbf{A}_{c 1}\right) \times \mathbf{u}_{p}\right|}
\end{array}\right.
$$

where, $\mathbf{A}_{c 1}$ is position vector of a point in cut axis.

During the process, the pinion generating rotate an angle $\theta_{c m}$, then the position vector and tangent vector in pinion generating can be expressed as

$$
\left\{\begin{array}{l}
\mathbf{A}_{\mathrm{cm}}=L\left[c_{p}, \theta_{c m}\right] \mathbf{A}_{\mathrm{cm}}+H \theta_{c m} \cdot \mathbf{c}_{p} \\
\boldsymbol{\tau}_{c m}=L \boldsymbol{\tau}_{c m}
\end{array}\right.
$$

So position vector and tangent vector in pinion can be expressed as

$$
\left\{\begin{array}{l}
\mathbf{r}_{1 \mathrm{~m}}=\mathbf{A}_{\mathrm{cm}}-\left(\mathbf{e}_{p} \cdot E_{m 1}+X_{g 1} \cdot \mathbf{p}\right) \\
\boldsymbol{\tau}_{1 m}=\boldsymbol{\tau}_{c m} \times \mathbf{p}
\end{array}\right.
$$

The normal vector can be expressed as

$$
\mathbf{n}_{1 m}=\boldsymbol{\tau}_{c m} \times \boldsymbol{\tau}_{1 m}
$$

The relative velocity can be expressed as

$$
\mathbf{V}_{1 c}=\left(\begin{array}{lll}
R_{a p} & \mathbf{p} & \mathbf{r}_{1 m}
\end{array}\right)+\left(\mathbf{c}_{p} \times \mathbf{A}_{c m}\right)+H \cdot \mathbf{c}_{p}
$$

Then meshing equation in the point expressed as

$$
\mathbf{V}_{1 c} \cdot \mathbf{n}_{1 m}=0
$$

The angle $\theta_{c m}$ can be obtained by solving Eq. (46), then the new root cone angle $\delta_{f 1}$ can be calculated as $\sin \delta_{f 1}=\mathbf{p} \cdot \mathbf{n}_{1 m}$ then the face cone angle should be modified. As the root cone angle is changed, to ensure the dedendum unchanged, $B$ should be adjusted. And position vector $\mathbf{A}_{c m}, \mathbf{A}_{c 1}$ can be obtained by Eq. (40) and Eq. (41).

Then the projection in the cut axis of vector formed between the point of middle of tool nose and the point in the cut axis (Fig.6) can be expressed as

$$
H_{1}=\left(\mathbf{A}_{c m}-\mathbf{A}_{c 1}\right) \cdot \mathbf{u}_{p}
$$

Similarly to $H_{2}$. Then pinion cut radius can be expressed as

$$
\left\{\begin{array}{l}
r_{c p 1}=\frac{R_{c 1}}{\cos \alpha_{11}}-H_{1} \tan \alpha_{11} \\
r_{c p 2}=\frac{R_{c 2}}{\cos \alpha_{12}}-H_{2} \tan \alpha_{12}
\end{array}\right.
$$

Vector of cut center in the coordinate system of pinion process can be expressed as

$$
\mathbf{S}_{p t}=\mathbf{A}_{c 1}-\left(E_{m 1} \cdot \mathbf{e}_{p}+X_{g 1} \cdot \mathbf{p}\right)+H_{1} \cdot \mathbf{u}_{p}
$$

Sliding base $X_{B 1}$ is calculated as

$$
X_{B 1}=\left(M\left(c_{p}, \theta_{c m}\right) \cdot \mathbf{S}_{p t}\right) \cdot \mathbf{c}_{p}+H \cdot \theta_{c m}
$$

As Fig. 5 shown, initial cradle angle can be expressed as

$$
\left\{\begin{array}{l}
L_{v}=-\mathbf{S}_{p t} \cdot \mathbf{e}_{p} \\
L_{\mathrm{h}}=\mathbf{S}_{p t} \cdot\left(\mathbf{e}_{p} \times \mathbf{c}_{p}\right)=\left(\mathbf{e}_{p}, \mathbf{c}_{p}, \mathbf{S}_{p t}\right) \\
q_{1}=a \tan \frac{L_{v}}{L_{h}}
\end{array}\right.
$$


Radial setting can be expressed as

$$
S_{r 1}=\frac{L_{v}}{\sin q_{1}}
$$

Tilt angle can be expressed as

$$
i_{1}=a \sin \left(\left|\mathbf{u}_{p} \times \mathbf{c}_{p}\right|\right)
$$

Spiral angle of pinion generating can be expressed as

$$
\beta_{c p}=a \tan \left(-\frac{\left(\mathbf{u}_{p}, \mathbf{c}_{p}, \mathbf{e}_{p}\right)}{\mathbf{e}_{p} \cdot \mathbf{u}_{p}}\right)
$$

Swivel angle can be expressed as

$$
j_{1}=q_{1}-\beta_{c p}
$$

\section{Establishment of TCA and ease-off model}

The mathematical mode of gear process is established in Fig.3. In particular, the pinion process have a motion of sliding base during the process, that is coordinate system $S_{d}$ have a motion relative to coordinate system $S_{P}$ along the direction of $Z_{P}$, and $X_{B 1}=X_{B 1}-H \cdot \varphi_{c}$. Similarly, the position vector and normal vector of pinion can be expressed as

$$
\left\{\begin{array}{l}
\mathbf{r}_{1}=f\left(u_{1}, \theta_{1}, \varphi_{c 1}\right) \\
\mathbf{n}_{1}=f\left(u_{1}, \theta_{1}, \varphi_{c 1}\right)
\end{array}\right.
$$

where, $u_{1}, \theta_{1}$ are the tooth surface parameters of pinion, and $\varphi_{c 1}$ is the cradle angle of pinion in process.

The model of mesh is built as Fig.7, $S_{h}$ is the mesh coordinate system, $S_{1}$ and $S_{2}$ is the coordinate system of pinion and gear, respectively. $S_{a}$ is an auxiliary coordinate system. $\phi_{1}$ and $\phi_{2}$ are the rotation angle of the pinion and gear from its initial generating position, respectively.

Then the position vector and normal vector of pinion and gear can be expressed in $S_{h}$ as

$$
\left\{\begin{array}{l}
\mathbf{r}_{h 1}\left(u_{1}, \theta_{1}, \varphi_{c 1}, \phi_{1}\right)=M_{h 1} \mathbf{r}_{1}\left(u_{1}, \theta_{1}, \varphi_{c 1}\right) \\
\mathbf{n}_{h 1}\left(u_{1}, \theta_{1}, \varphi_{c 1}, \phi_{1}\right)=L_{h 1} \mathbf{n}_{1}\left(u_{1}, \theta_{1}, \varphi_{c 1}\right) \\
\mathbf{r}_{h 2}\left(u_{2}, \theta_{2}, \varphi_{c 2}, \phi_{2}\right)=M_{h a} M_{a 2} \mathbf{r}_{2}\left(u_{2}, \theta_{2}, \varphi_{c 2}\right) \\
\mathbf{n}_{h 2}\left(u_{2}, \theta_{2}, \varphi_{c 2}, \phi_{2}\right)=L_{h a} L_{a 2} \mathbf{n}_{2}\left(u_{2}, \theta_{2}, \varphi_{c 2}\right)
\end{array}\right.
$$

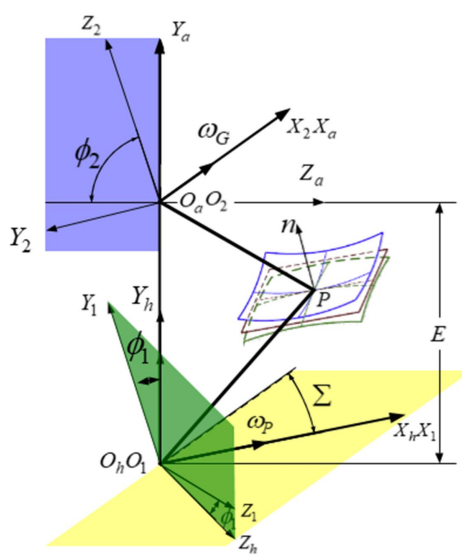

Fig.7 Coordinate system of meshing

In mesh point, the equations are satisfied 


$$
\left\{\begin{array}{l}
\mathbf{r}_{h 1}\left(u_{1}, \theta_{1}, \varphi_{c 1}, \phi_{1}\right)=\mathbf{r}_{h 2}\left(u_{2}, \theta_{2}, \varphi_{c 2}, \phi_{2}\right) \\
\mathbf{n}_{h 1}\left(u_{1}, \theta_{1}, \varphi_{c 1}, \phi_{1}\right)=\mathbf{n}_{h 2}\left(u_{2}, \theta_{2}, \varphi_{c 2}, \phi_{2}\right)
\end{array}\right.
$$

Solving Eq. (58), a series of mesh points can be obtained. According to the parameters of mesh points, the contact pattern and transmission can be achieved.

Ease-off is the deviation from conjugation between two tooth surfaces from a pair of mating members in contact. In this paper, we take the deviations between conjugate tooth surface of gear and pinion tooth surface to draw ease-off topography.

The conjugate gear tooth surface can be deduced as

$$
\left\{\begin{array}{l}
\mathbf{r}_{1 \mathrm{c}}=M_{12}\left(\phi_{2}\right) \mathbf{r}_{2}\left(u_{2}, \theta_{2}, \varphi_{c 2}\right) \\
\mathbf{n}_{1 c}=L_{12}\left(\phi_{2}\right) \mathbf{n}_{2}\left(u_{2}, \theta_{2}, \varphi_{c 2}\right) \\
f_{2 c}\left(u_{2}, \theta_{2}, \varphi_{c 2}\right)=\frac{\partial \mathbf{r}_{1 \mathrm{c}}}{\partial \mathbf{n}_{1 c}} \cdot \mathbf{n}_{1 c}=0
\end{array}\right.
$$

Then the deviations can be calculated as

$$
\left\{\begin{array}{l}
\Delta=\left[\mathbf{r}_{1}\left(u_{1}, \theta_{1}, \varphi_{c 1}\right)-\mathbf{r}_{1 \mathrm{c}}\left(u_{2}, \theta_{2}, \phi_{2}, \varphi_{c 2}\right)\right] \cdot \mathbf{n}_{1}\left(u_{1}, \theta_{1}, \varphi_{c 1}\right) \\
f_{1 c}\left(u_{1}, \theta_{1}, \varphi_{c 1}\right)=0
\end{array}\right.
$$

\section{Numerical example and cut experiment}

In order to verify the feasibility of method proposed in this paper, an example is illustrated. The basic parameters of spiral bevel gear are listed in Table 1 , and machine setting parameters corresponding to the contact path of $45^{\circ} 、 60^{\circ}$ 、

\begin{tabular}{|c|c|c|}
\hline Parameter & Gear & Pinion \\
\hline Shaft angle $\sum /\left(^{\circ}\right)$ & \multicolumn{2}{|c|}{90} \\
\hline Modulo $m /(\mathrm{mm})$ & \multicolumn{2}{|c|}{7.16} \\
\hline Spiral angle $\beta /\left(^{\circ}\right)$ & \multicolumn{2}{|c|}{35} \\
\hline Pressure angle $\alpha_{0} /\left(^{\circ}\right)$ & \multicolumn{2}{|c|}{20} \\
\hline Face width $b /(\mathrm{mm})$ & \multicolumn{2}{|c|}{45} \\
\hline Number of tooth $z$ & 35 & 18 \\
\hline Addendum $h_{a} /(\mathrm{mm})$ & 3.55 & 8.78 \\
\hline Dedendum $h_{f} /(\mathrm{mm})$ & 10.26 & 5.03 \\
\hline
\end{tabular}
$70^{\circ}$ are calculated according to the method proposed in this paper and listed in Table 2.

Table 1 basic parameters

\begin{tabular}{|c|c|c|c|c|}
\hline \multirow{2}{*}{ Parameter name } & \multirow{2}{*}{ Gear } & \multicolumn{3}{|c|}{ Pinion } \\
\hline & & (a) $\eta=45^{\circ}$ & (b) $\eta=60^{\circ}$ & (C) $\eta=70^{\circ}$ \\
\hline Tilt angle $i /\left(^{\circ}\right)$ & 0.50 & 2.24 & 3.34 & 4.67 \\
\hline Swivel angle $j /\left(^{\circ}\right)$ & 25.72 & 136.97 & 178.80 & 204.21 \\
\hline Radial setting $S_{r} /(\mathrm{mm})$ & 107.3339 & 106.7915 & 105.9273 & 104.6725 \\
\hline Initial cradle angle $q /\left(^{\circ}\right)$ & 60.72 & 60.60 & 59.49 & 58.52 \\
\hline Vertical offset $E_{m} /(\mathrm{mm})$ & 0 & -0.3235 & -0.5711 & -0.8177 \\
\hline Sliding base $X_{B} /(\mathrm{mm})$ & -0.3193 & -2.3564 & 0.6752 & -4.9013 \\
\hline Helical motion velocity coefficient $H /(\mathrm{mm} / \mathrm{rad})$ & 0 & 2.5913 & -1.7987 & -6.2838 \\
\hline Machine root angle $\gamma /\left(^{\circ}\right)$ & 58.34 & 27.07 & 27.80 & 27.56 \\
\hline Machine center to cross point $X_{g} /(\mathrm{mm})$ & 0 & -0.0123 & -0.6516 & -1.6552 \\
\hline Ratio of roll $R_{a p}$ & 1.121120 & 2.181756 & 2.165372 & 2.141237 \\
\hline
\end{tabular}

Table 2 machine setting parameters 
Geng, Xiaozhong Deng, Jing Deng, Jiang and Han,

Journal of Advanced Mechanical Design, Systems, and Manufacturing, Vol.15, No.1 (2021)

The results of tooth contact analysis based on the parameters listed in Table 2 are showed in Fig.7.

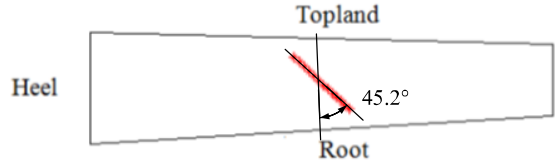

(a) Contact path of gear convex side

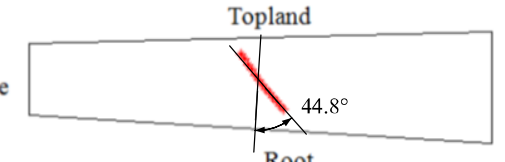

(b) Contact path of gear concave side

\section{Heel}

(a) Contact path corresponding to plan $\mathrm{A}$

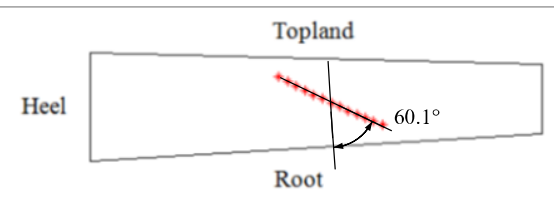

(a) Contact path of gear convex side

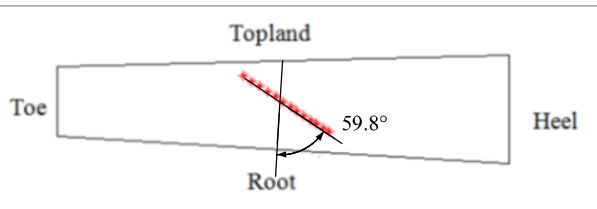

(b) Contact path of gear concave side

(b) Contact path corresponding to plan B

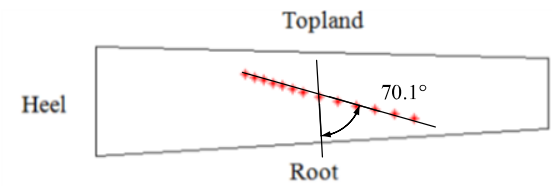

(a) Contact path of gear convex side

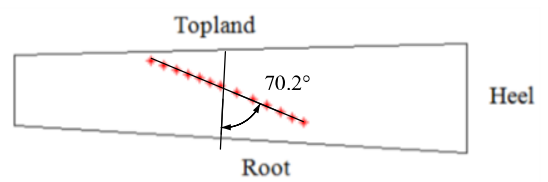

(b) Contact path of gear concave side

(c) Contact path corresponding to plan $\mathrm{C}$

Fig. 8 Results of contact path

As Fig. 8 shown, (a) the contact path of convex is $45.2^{\circ}$ and the concave is $44.8^{\circ}$, there are errors with predesigned by $1 \%$ and $0.4 \%$, respectively; (b) the contact path of convex is $60.1^{\circ}$ and the concave is $59.8^{\circ}$, there are errors with predesigned by $0.16 \%$ and $0.33 \%$, respectively; (c) the contact path of convex is $70.1^{\circ}$ and the concave is $70.2^{\circ}$, there are errors with predesigned by $0.14 \%$ and $0.28 \%$, respectively. In conclusion, the errors are less than $0.5 \%$, and the results are accords with the predesigned, and therefore proved the proposed method is effective.

Finally a cut experiment is carried out on Gleason600HC according to parameters of (c). The results of TCA and ease-off topography are in Fig.9.

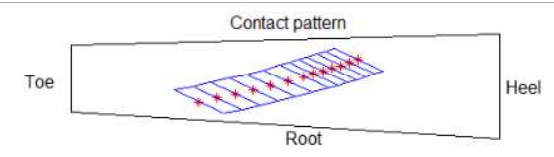

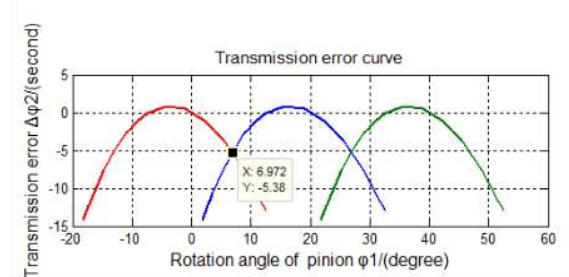

(a) Contact pattern and transmission error of gear convex side Contact pattern
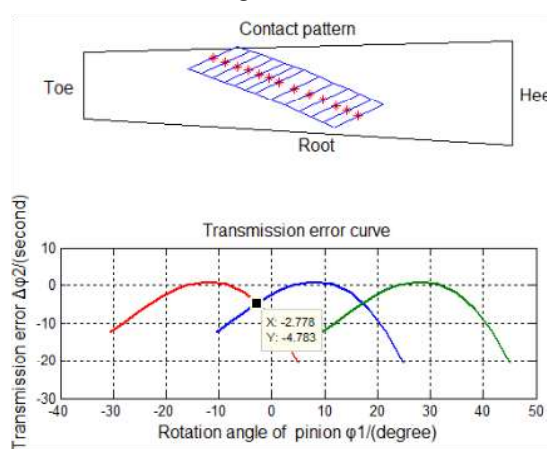

(c) Contact pattern and transmission error of gear concave side

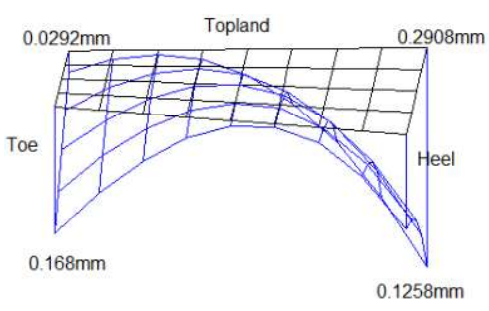

(b) Ease-off topography of pinion concave side

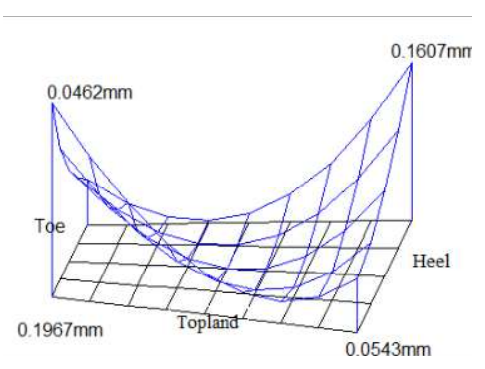

(d) Ease-off topography of pinion convex side

Fig.9 Results of TCA and ease-off topography 
As Fig.9 illustrate, (a) is contact pattern and transmission error of gear convex side; (b) is ease-off of pinion concave side; (c) is contact pattern and transmission error of gear concave side; (d) is ease-off of pinion convex side. The contact pattern is in the middle of tooth surface, and transmission error is stable. Comparing (a) and (b), the contact pattern is consistent, which is bias in from the topland of heel to the root of the toe in gear convex side; similarly to (c) and (d), while the contact pattern is from the root of heel to the topland of the toe in gear concave side. The curve of transmission error is smooth and continuous. The transmission error is 5.38 second and 4.783 second for convex side and concave side of gear.

The process of pinion is as shown in Fig. 10.

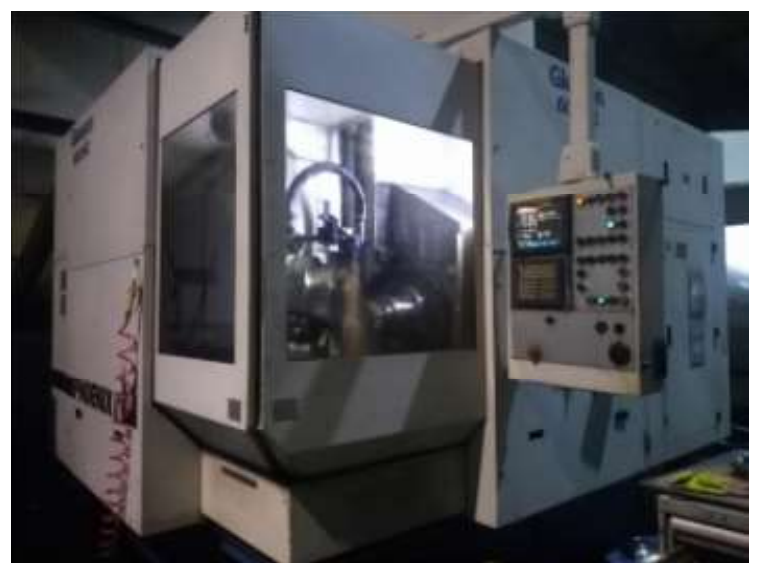

Fig.10 Process of pinion

After pinion is processed, the measure result of pinion is showed in Fig.11.

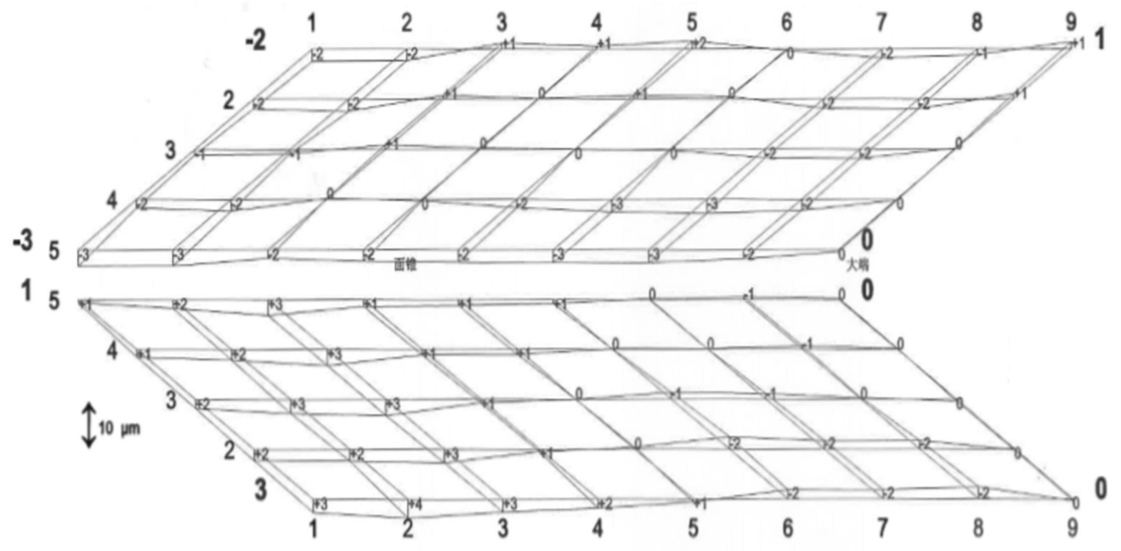

Fig.11 Result of pinion measure

The measurement of pinion tooth surface between theoretical and process are as shown in Fig.11, for concave side, the biggest error is $3 \mu \mathrm{m}$ at the topland of toe, and for convex side, the biggest error is $4 \mu \mathrm{m}$ at the root of toe. The errors may be caused by machine tool error, workpiece error, installation error and so on. While the errors meet the engineering accuracy requirements.

The compare of time costing in pinion process by five-cut method and duplex helical method are listed in Table 3.

Table 3 Compare of time costing in pinion process

\begin{tabular}{cccc}
\hline Time costing in finish cut & Concave side & Convex side & Total \\
\hline Five-cut method & 25.3 second & 26 second & 51.3 second \\
Duplex helical method & 53.3 second & 53.3 second \\
\hline
\end{tabular}

For time costing in pinion process, concave side and convex side are 25.3 second and 26 second respectively. The time of finish cut is 51.3 second which is nearly equivalent to the time costing in this paper (53.3 second). While the time cost of rough cut and multiple installations by five-cut method are not considered. So the duplex helical method is 
higher efficiency.

Finally a rolling test is carried out. The pair runs smoothly without obvious noise and the results of contact patterns are in Fig. 12.

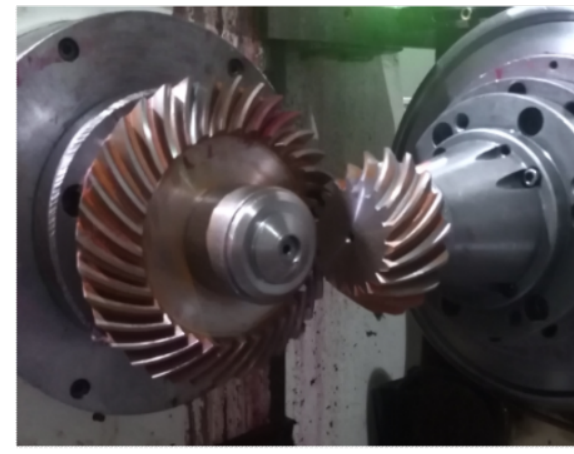

(a) Rolling test

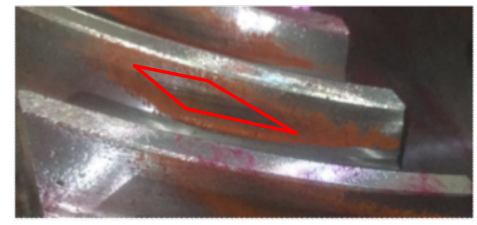

(b) Convex side

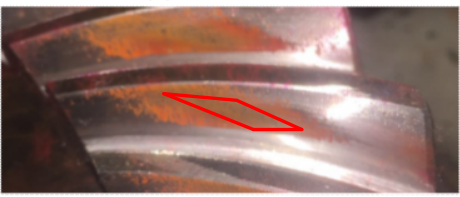

(c) Concave side

Fig.12 Result of rolling test

As Fig.12 display, (a) is the scene of roiling tests; (b) is real contact pattern for gear convex; (c) is real contact pattern for gear concave. The contact patterns are bias in, and account for one third of tooth surface. Comparing the rolling test (Fig.12) to the results of TCA (Fig.9), the contact patterns are accord with from the point of the position and shape. Application example shows the effectiveness and feasibility of the method.

\section{Conclusion}

The machine setting parameters which guarantee contact path are calculated by calculating relative curvatures along the direction of contact path and its perpendicular.

A model of tooth contact analysis is established. And numerical examples are calculated by the method. The results of TCA have a difference less than $0.5 \%$ compare to the value of predesigned.

A cut experiment is carried out, the rolling test are in accord with the results of TCA. Finally, the results show the correctness and the affectivity of the proposed method.

\section{Acknowledgments}

The authors would like to thank the financial aid and support from the National Natural Science Foundation of China (Grant NO. 51475141, Grant NO. 51705134, and Grant NO. 51705135). We are grateful to the reviewers and editors for their valuable comments and suggestions.

\section{Reference}

Cao, X.M., Deng, X.Z., Wei, B.Y., A novel method for gear tooth contact analysis and experimental validation, Mechanism and Machine Theory, Vol.126 (2018), DOI:10.1016/j.mechmachtheory.2018.04.002

Cao, X.M., Fang, Z.D., Zhang, J.L., Function-oriented active tooth surface design of spiral bevel gears, Chinese Journal of Mechanical Engineering, Vol.44, No. 8 (2008), DOI: 10.3901/JME.2008.07.209 (in Chinese)

Fan Q., Ease-Off and application in tooth contact analysis for face-milled and face-hobbed spiral bevel and hypoid gears, Theory and Practice of Gearing and Transmissions, Mechanisms and Machine Science, Vol.34 (2016), DOI: 10.1007/978-3-319-19740-1_15

Fan, Q., Enhanced algorithms of contact simulation for hypoid gear drives produced by face-milling and face-hobbing processes, Journal of Mechanical Design, Vol.129, No. 1 (2007), DOI: 10.1115/1.2359475

Gleason Works, Calculation instructions generated hypoid gears duplex helical method, the Gleason Works, (1971), New York

Gonzalez-Perez, A. Fuentes, K. Hayasaka, Computerized design and tooth contact analysis of spiral bevel gears 
generated by the duplex helical method, ASME 2011 International Design Engineering Technical Conferences and Computers and Information in Engineering Conference, Vol.8 (2011), DOI: 10.1115/DETC2011-47108

Stadtfeld H.J., Tribology aspects in angular transmission systems part IV: spiral bevel gears, Gear Technology, 66-72(2011).

Kolivand, M., Kahraman, A., An Ease-Off based method for loaded tooth contact analysis of hypoid gears having local and global surface deviations, Journal of Mechanical Design, Vol.132, No.7(2010), DOI: 10.1115/1.4001722

Litvin, F.L., Gutman Y., Methods of synthesis and analysis for hypoid gear drives of "formate" and "helixform", Journal of Mechanical Design, Vol.103, No.4 (1981), pp.83-110.

Litvin, F.L., Fuentes, A., "Gear geometry and applied theory”, 2nd edition, Cambridge University Press, (2004), New York

Litvin, F.L., Zhang, Y., Local synthesis and tooth contact analysis of face-milled spiral bevel gears, NASA CR4342 (1991), pp.48-68.

Litvin F.L., Fuentes A., Hayasaka K., Design, manufacture, stress analysis, and experimental tests of low-noise high endurance spiral bevel gears, Mechanism and Machine Theory, Vol.41, No.1, (2006), DOI: 10.1016/j.mechmachtheory

Litvin F.L., Zhang Y., Lundy M., C. Heine, Determination of settings of a tilted head cutter for generation of hypoid and spiral bevel gears, Journal of Mechanisms, Transmissions, and Automation in design, Vol.110, No.4 (1988), DOI: $10.1115 / 1.3258950$.

Nie, S.W.., Deng, J., Deng, X.Z., A flank modification method for spiral bevel gears based on mismatch topography adjustment, Journal of Advanced Mechanical Design, Systems, and Manufacturing, Vol.12, No.2 (2018), DOI:10.1299/jamdsm.2018jamdsm0057

Shtipelman, B.A., Design and Manufacture of Hypoid Gears, A Wiley-Interscience Publication, (1978), New York

Shih,Y.P, A novel ease-off flank modification methodology for spiral bevel and hypoid gears, Mechanism and Machine Theory, Vol.45, No.8 (2010), DOI: 10.1016/j.mechmachtheory.2010.03.010

Tsay, C.B., Lin, J.Y., A mathematical model for the tooth geometry of hypoid gears, Mathematical and Computer Modelling, Vol.18 (1993), pp.23-34

Yan Z.D., Mathematical of gear meshing theory, Mathematics in Practice and Theory, Vol.3 (1980), pp.1-9 (in Chinese)

Zhang W.Q., Ma P.P., Guo X.D., Contact characteristics control method of spiral bevel gears based on ease-off, Journal of Beijing University of Technology, Vol.47(2018), pp.1024-1031. (in Chinese)

Zhang, Y., Yan, H.Z., New methodology for determining basic machine settings of spiral bevel and hypoid gears manufactured by duplex helical method. Mechanism and Machine Theory, Vol.100 (2016), DOI: 10.1016/j.mechmachtheory.2016.02.015 